\title{
Laser powder bed fusion in high-pressure atmospheres
}

\author{
P. Bidare ${ }^{1}$ (D) I. Bitharas ${ }^{1} \cdot$ R. M. Ward ${ }^{2} \cdot$ M. M. Attallah ${ }^{2} \cdot$ A. J. Moore ${ }^{1}$
}

Received: 10 May 2018 / Accepted: 18 July 2018 / Published online: 7 August 2018

(C) The Author(s) 2018

\begin{abstract}
High-speed imaging and schlieren imaging were used to investigate the interaction of the laser beam with the powder bed at pressures up to 5 bar, in argon and helium atmospheres. The entrainment of powder particles in the flow of shielding gas generated by the laser plume, and hence denudation, was reduced at high pressure for both gases. However, for argon, high pressure increased the temperature of both the melt pool and the laser plume, which significantly increased the generation of spatter and ionisation of the metal vapour with degraded surface smoothness and continuity. For helium, the formation of spatter and plasma did not increase with the increase in pressure above that observed at atmospheric pressure: its higher thermal conductivity and thermal diffusivity limited the laser plume temperature. Layers built at 5 bar in helium had a surface smoothness and continuity comparable to those built in argon at atmospheric pressure, but achieved at a higher laser scan speed, suggesting that a high-pressure helium atmosphere may be used to enhance the build rate.
\end{abstract}

Keywords High-speed imaging $\cdot$ Laser powder bed fusion $\cdot$ High pressure atmosphere $\cdot$ Argon atmosphere $\cdot$ Helium atmosphere

\section{Introduction}

In laser powder bed fusion (PBF), a focussed laser beam selectively fuses regions of a powder bed in order to build metal components layer-by-layer [1]. An inert atmosphere, typically argon, is used at or very close to atmospheric pressure. Production components can be manufactured by commercial PBF systems, but thermally induced residual stresses and defects remain an issue. Hence, understanding and improving the PBF process is an active area of research to increase reliability and productivity.

Recent studies in PBF at atmospheric pressure have shown that the laser plume of metal vapour and plasma from the melt pool induces a flow in the shielding gas, which entrains powder particles towards the track [2-4]. The resulting depletion

Electronic supplementary material The online version of this article (https://doi.org/10.1007/s00170-018-2495-7) contains supplementary material, which is available to all users.

\section{P. Bidare}

p.bidare@hw.ac.uk; bidarep@gmail.com

1 Heriot-Watt University, Institute of Photonics and Quantum Sciences, Edinburgh EH14 4AS, UK

2 School of Metallurgy and Materials, University of Birmingham, Birmingham B15 2TT, UK of powder (denudation) increases the likelihood of porosity and the surface roughness of built parts. If the ambient pressure is reduced, the speed of the laser plume increases $[2,5]$. In the hydrodynamic flow regime, the associated increase in the induced flow entrains more powder particles, and particles from further away on the powder bed, towards the laser scan line, increasing the width of the denuded zone. As the pressure is further reduced into the transition region between hydrodynamic and molecular flow, particles are still entrained from further away on the powder bed than at atmospheric pressure. However, these particles do not reach the melt pool due to the increased speed and wider ejection angle of the laser plume: profiles and cross-sections of the track reveal a drastic reduction in its cross-sectional area. Eventually, at pressures low enough to reach the molecular flow regime, there is no entrainment of particles towards the laser scan line and all particles are repelled from the melt pool by the expansion of the laser plume [2, 5].

An increase in ambient pressure might reduce denudation and porosity: the reduced velocity of the laser plume and the resulting flow induced in the shielding gas might cause fewer powder particles to be entrained. The reduced recoil pressure from the laser plume at high pressure might lead to a more stable melt pool and a smoother build. It might also reduce condensation coating on the machine windows from evaporation of metal from the melt pool and selective evaporation $[6,7]$ 
of low boiling temperature elements within an alloy. Gieseke et al. [8] proposed that laser PBF at high pressure might be advantageous for metals and metallic alloys with low boiling temperatures and evaporation heats. Specifically for magnesium, it was suggested that the excessive vaporisation that prevented a three-dimensional build at atmospheric pressure (boiling point $1093{ }^{\circ} \mathrm{C}$ ) might be eliminated at 3-bar absolute pressure (boiling point increased to $1220^{\circ} \mathrm{C}$ ). However, this suggestion was not implemented. To the best of the authors' knowledge, there are no reports in the literature of laser PBF significantly above atmospheric pressure.

In this paper, we investigate the laser interaction with a single powder layer at high pressures in order to understand the process conditions that might enable multiple layers to be built in the future. We report the penetration depth obtained in order to gain further insight into the process. Finally, we discuss the implication of our findings for potential PBF at high pressure.

\section{Experimental system}

We previously reported the design and characterisation of an open-architecture PBF system for in situ measurements [9]. The PBF system is computer controlled for the automated build of fully dense components, enabling in-process measurements under realistic build conditions. However, for this study, the laser interaction with a single powder layer was investigated in order to understand the process conditions at high pressures that might enable multiple layers to be built in the future.

For the work presented here, the open-architecture PBF system was encased in a custom-made pressure chamber, Fig. 1. The pressure chamber was essentially the same as a vacuum chamber used to perform PBF at sub-atmospheric pressures down to $10 \mu$ bar [5] and so it is not described in detail again here. The key difference was that the high-vacuum viewport assemblies, which were used to provide optical access to the powder bed, were reinforced externally with stainless steel adapter plates and additional O-rings to prevent them from blowing out under high pressure. The top of the pressure chamber contained a viewport for the PBF laser, and two symmetrical viewports for white light illumination (shown in the figure) and imaging of the powder bed (not shown). For this study, two additional viewports (Thorlabs VPCH42-C without antirefection coatings) in the ends of the chamber were used for direct imaging and schlieren imaging.

The pressure chamber was connected directly to the shielding gas cylinder through a non-return inlet valve. For argon, the chamber was purged by continuously filling for $10 \mathrm{~min}$, with the pressure gauge port in the top of the chamber intentionally opened, to reduce the $\mathrm{O}_{2}$ concentration to < $0.1 \%$. The pressure gauge was then tightened in position and the chamber pressure increased to the required value. For helium, the process was reversed: the pressure chamber was filled from the top in the initial purging process so that the heavier air was displaced via the bottom of the chamber. The viewport windows were calculated to withstand 10 bar, although the highest pressure tested in these experiments was 5 bar. The pressure decrease due to leakage was negligible during the time required to scan the laser tracks and islands at a given pressure.

Experiments were performed on gas-atomised stainless steel $316 \mathrm{~L}$ powder (Renishaw PLC) with particle diameters in the range 15 to $45 \mu \mathrm{m}$ and a mean diameter of $30 \mu \mathrm{m}$ [10]. Powder layers of thickness $50 \mu \mathrm{m}$ were spread on stainless steel 304-L build plates (coupons), which had been roughened by manual, circular rubbing with P400 sandpaper. The powder
Fig. 1 Schematic of the openarchitecture PBF system with pressure chamber

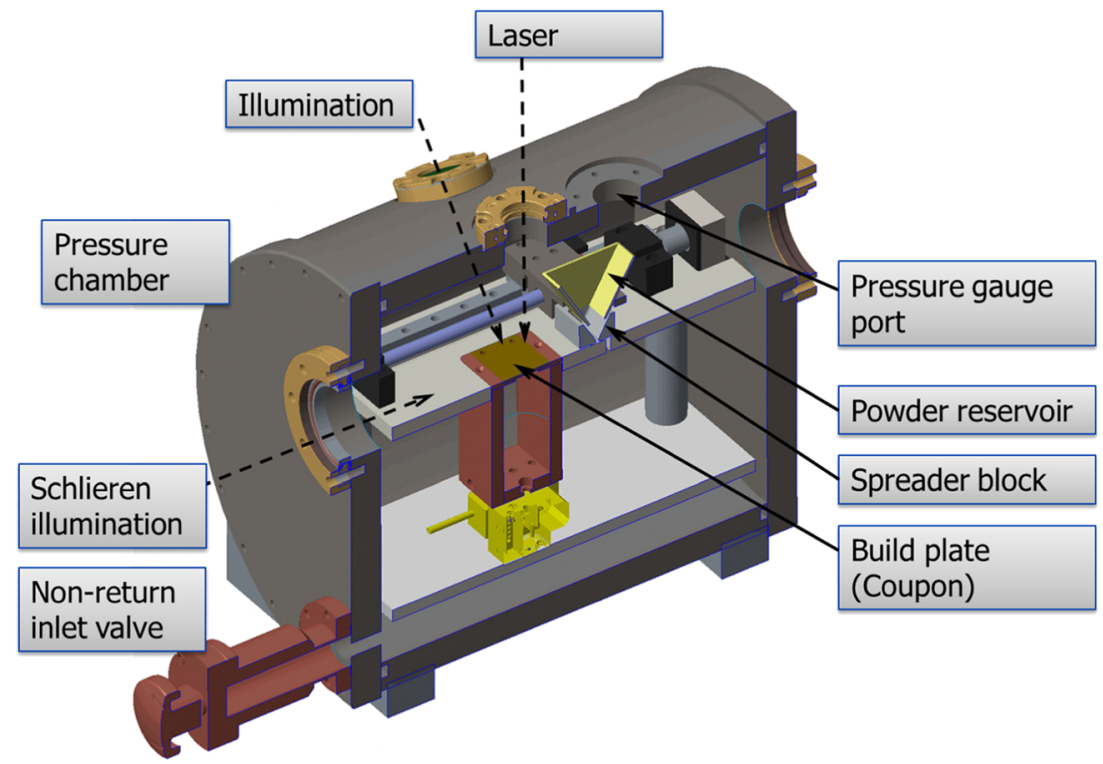


was melted with a single-mode fibre laser $(1070 \mathrm{~nm})$ focussed to a spot of diameter $50 \mu \mathrm{m}$ [9].

High-speed image sequences were recorded with a Phantom V2512 monochrome camera at 40,000 frames per second (fps) and $768 \times 368$ pixels resolution, with illumination of the powder bed by a Lumencor SOLA SM white light source [5]. The camera was fitted with a band-stop filter to block light from the PBF laser. A 'top' view of the powder bed was obtained through the viewport in the top of the pressure chamber with the camera angled at $\sim 20^{\circ}$ to the vertical. A 'side' view was obtained through one of the viewports in the side of the pressure chamber with the camera at $\sim 10^{\circ}$ to the horizontal.

A portable z-type schlieren system [11] used a 300-W tungsten lamp to introduce collimated white light illumination across the powder bed through the two end viewports [4]. Images were recorded with the high-speed camera using a variable focus telephoto lens (focal length $200-500 \mathrm{~mm}$ ) at $80,000 \mathrm{fps}$ and $384 \times 512$ pixels resolution. The playback speed for the schlieren results has been adjusted to produce the same apparent time dilation as for the direct imaging videos. For the schlieren experiments, the camera was fitted with a polariser to remove glare in addition to the band-stop filter; the powder layer was spread on the coupon and the spreader block assembly was then carefully removed so that it did not obstruct the collimated schlieren illumination passing across the powder bed.

\section{Results}

Results were recorded for three different laser power and scan speed combinations that provided the same line energy (laser power divided by scan speed) of $250 \mathrm{~J} / \mathrm{m}: 50 \mathrm{~W}$ and $0.2 \mathrm{~m} / \mathrm{s}$, $100 \mathrm{~W}$ and $0.4 \mathrm{~m} / \mathrm{s}$ and $200 \mathrm{~W}$ and $0.8 \mathrm{~m} / \mathrm{s}$. The $100-\mathrm{W}$ laser power condition has been shown to build parts with $>99 \%$ density in our system [9] at atmospheric pressure (1 bar). Single tracks and rectangular islands were scanned at absolute pressures of 1, 3 and 5 bar. An island comprised of an individual area of $1 \times 2 \mathrm{~mm}^{2}$ scanned by multiple adjacent tracks: the scan spacing was $50 \mu \mathrm{m}$ between adjacent tracks and the laser was switched off for $\sim 500 \mu$ s at the end of each line.

\subsection{Argon atmosphere}

Figure 2 and Video Fig. 2 show direct imaging for top views of the powder bed for single track scans, recorded with increasing pressure in argon. Concentrating on the $100 \mathrm{~W}$ and $0.4 \mathrm{~m} / \mathrm{s}$ condition as typical of a good build condition, the results at 1 bar in Fig. 2a were consistent with our previous observations $[4,5]$. The laser plume points vertically upwards and induces a flow in the shielding gas that entrains particles from all directions on the powder bed. Entrained powder particles are either consolidated into the track or ejected upwards. Some of these particles are incandescent due to their
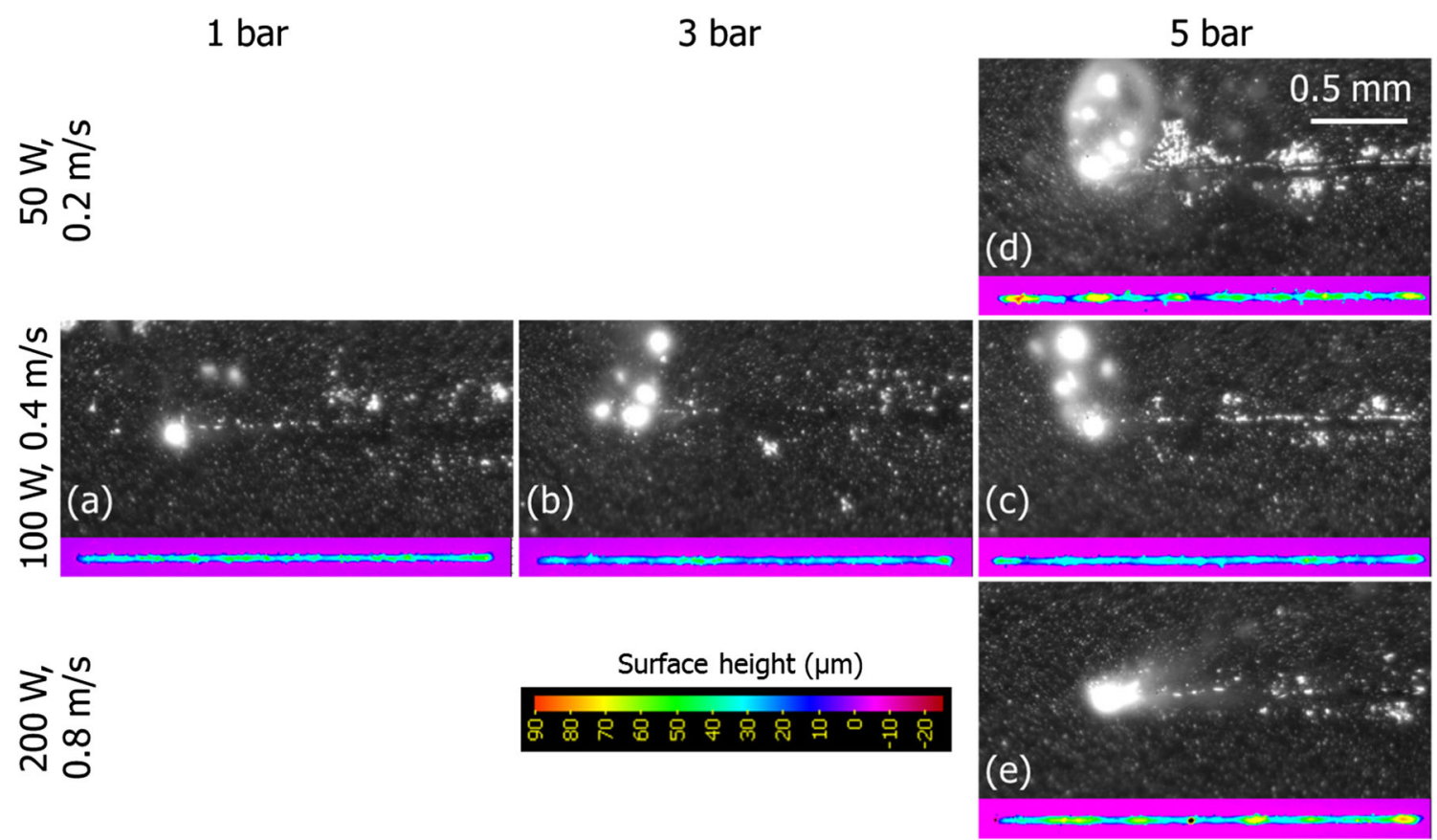

Fig. 2 High-speed images for top views of the powder bed in argon when scanning single tracks at the pressures and process settings (laser power and scan speed) indicated. Scan direction is right to left. Denudation is reduced as the pressure increases at a laser power of $100 \mathrm{~W}$ and scan speed of $0.4 \mathrm{~m} / \mathrm{s}$; the formation of plasma and spatter increases as the laser power and scan speed are increased at 5 bar. The videos for all figures are included in the supplementary material with the online version of this paper. Inset is the height profile for the entire length $(5 \mathrm{~mm})$ of each bead, i.e. not to the same scale as the image 
interaction with the laser beam or the laser plume. The laser plume also ejects incandescent spatter due to instabilities in the melt pool.

At 3 and 5 bar, Fig. $2 b, c$, the powder particle entrainment and the resulting denudation do indeed decrease as the pressure increases. However, the amount of plasma in the laser plume above the melt pool increases significantly as the pressure increases. The amount of molten spatter and the size of the spatter particles also both increase. Plasma can even be seen around some of these spatter particles, particularly at 5 bar, which suggests that the spatter is at a high enough temperature to produce metal vapour (an effect observed even at ambient pressure, similar to the Leidenfrost effect) which is then ionised via the transfer of thermal energy from the plume. This increased ionisation indicates that the temperature of the laser plume increases at high pressure. These observations are discussed in Section 4.

Figure $2 \mathrm{~d}$, c, e shows the effect of changing the process setting at 5 bar. The $50 \mathrm{~W}$ and $200 \mathrm{~W}$ conditions are consistent with our previous observations $[4,5]$ where the laser plume points forwards and backwards respectively with respect to the laser scan direction. In all cases, the denudation at 5 bar was less than the corresponding process setting at 1 bar; however, there was more plasma, and more and larger spatter particles were generated. Less plasma was produced at the
$200 \mathrm{~W}$ condition than at 50 and $100 \mathrm{~W}$ at 5 bar: the higher scan speed and backwards direction of the laser plume meant that the incident laser beam had less time to interact with the laser plume. Many of the spatter particles produced at $200 \mathrm{~W}$ do not produce vapour or plasma, for the same reason.

The build height measured relative to the build plate for the single track scans is inset in Fig. 2. These bead profiles are for the full 5-mm scan length of the track and are therefore at a different scale to the images in the figure. In general, the smoothness and continuity of the track worsened with an increase in pressure at a given laser power and scan speed, due to an increase in absorption, scattering and shadowing of the incident laser beam. Generally, the smoothness and continuity of the track improved with an increase in laser power and scan speed at high pressure. These effects are discussed in Section 4.

Figure 3 and Video Fig. 3 show direct imaging for side views of the powder bed for single track scans, at 1 and 5 bar in argon. Although the contrast between the incandescent spatter and cold particles is worse in the side images than for the top images, because the illumination direction is less favourable, the cold particles can still be discerned. The results at 1 bar, Fig. 3a, c, for both 100 and $200 \mathrm{~W}$, are consistent with our previous observations $[4,5]$. The laser plume contains metal vapour, which can be observed directly when it is ionised. The flow in the shielding gas induced by the laser
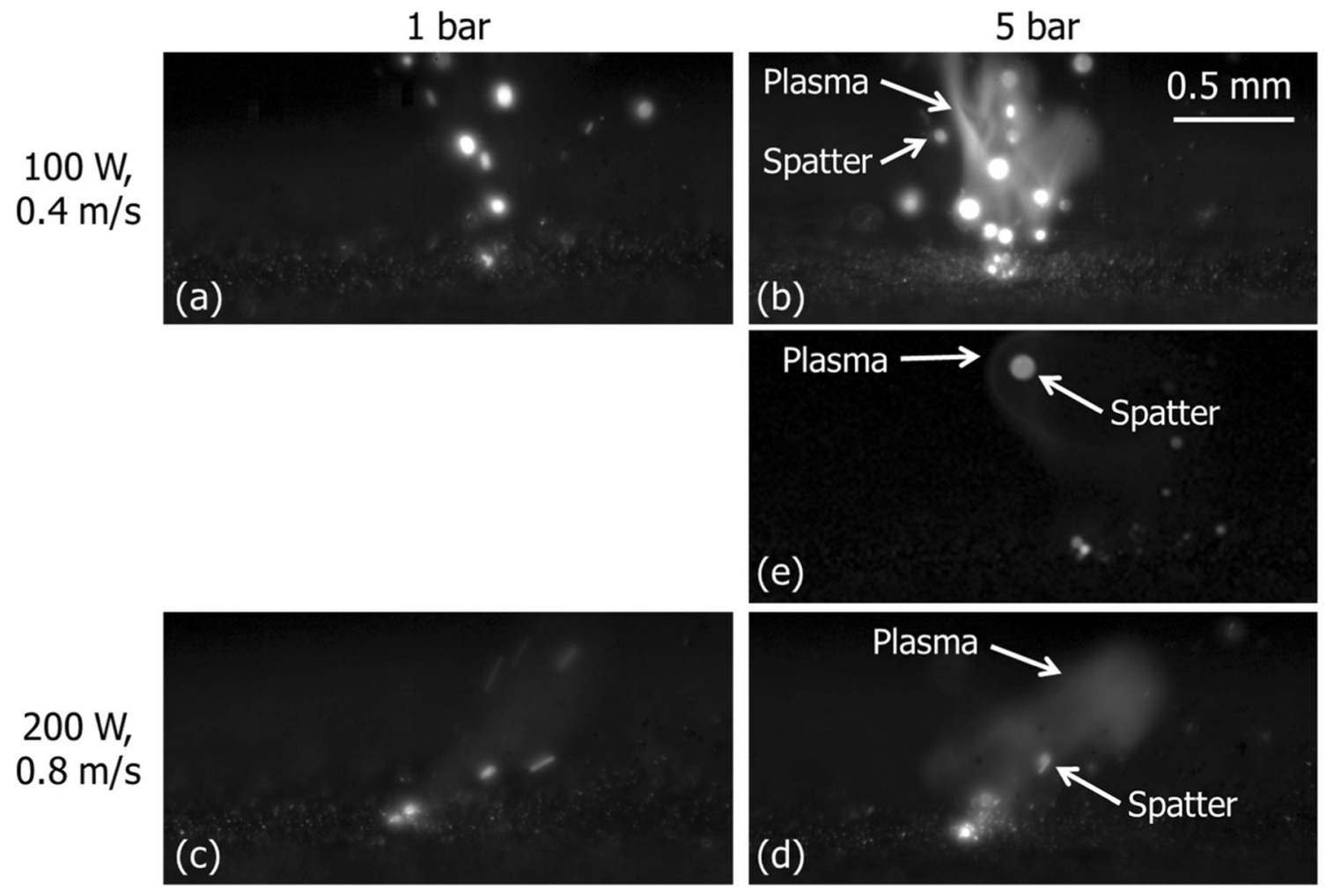

Fig. 3 High-speed images for side views of the powder bed in argon when scanning single tracks at the pressures and process settings indicated. Scan direction is right to left. The formation of plasma and spatter increases as the laser power is increased at 5 bar; less plasma and spatter was formed at the higher scan speed due to less interaction between the laser and the laser plume. An additional band-pass filter at $632 \pm 10 \mathrm{~nm}$ was included for Fig. 3e, in order to image the incandescent spatter more clearly 
plume entrains individual powder particles, which can become airborne: some particles are incandescent due to their interaction with the laser beam or the laser plume. Powder agglomerates are also entrained and some can be melted by the laser beam to produce incandescent spatter. The laser plume also ejects spatter due to instabilities in the melt pool. At 5 bar, Fig. 3b, d, the amount of plasma and number of powder agglomerates increased compared to $1 \mathrm{bar}$, indicating an increased process temperature. The amount and average size of molten spatter therefore increased at high pressure. As noted above, the amount of plasma and spatter was less for $200 \mathrm{~W}$ than for $100 \mathrm{~W}$ at 5 bar, because the interaction time of the laser beam with the laser plume was reduced. Figure $3 \mathrm{e}$ shows the $100 \mathrm{~W}$ condition at 5 bar with an additional bandpass filter at $632 \pm 10 \mathrm{~nm}$ inserted in front of the camera lens, to reduce saturation in the image from incandescent spatter particles. The plasma produced by the molten spatter can be clearly seen in the video. Without saturation in the image, the different intensities of the spatter particles themselves can be observed, indicating that they are produced at different temperatures.

Figure 4 and Video Fig. 4 show high-speed schlieren imaging for single track scans with the laser travelling towards the camera. It is not possible to resolve either individual cold powder particles, or the laser plume immediately above the melt pool, due to the lower magnification than that used for the direct imaging. However, the thermal plume of heated shielding gas and metal vapour rising above the melted track, as well as spatter and heated particles, can be seen. The images in Fig. 4 were taken with the laser spot at the same distance from the start of the scan line $(4 \mathrm{~mm})$ so as to compare the evolution of the thermal plume. At higher laser scan speeds, less time is required to scan $4 \mathrm{~mm}$ and so the plume rises less far above the powder bed. At high pressure, the thermal plume
Fig. 4 High-speed schlieren images during single track scans towards the camera at the pressures and process settings indicated. In each case, the schlieren sensitivity is the same and the image is taken when the laser had scanned $4 \mathrm{~mm}$ on the powder bed surface. At high pressure, the thermal plume rises less due to the reduced velocity of the laser plume; the schlieren image features are darker due to the increased temperature of the plume
1 bar

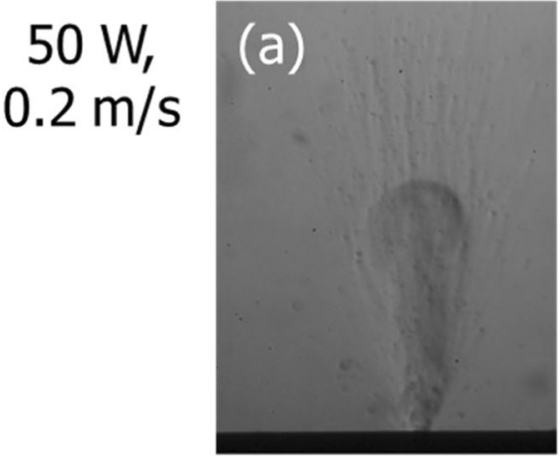

\section{$100 \mathrm{~W}$, $0.4 \mathrm{~m} / \mathrm{s}$}

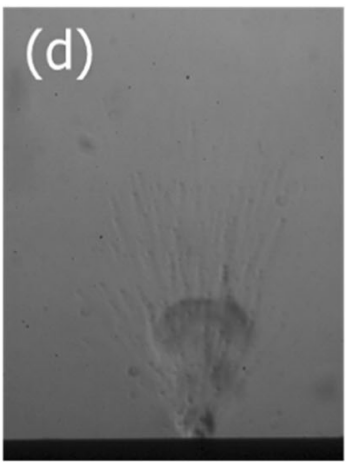

$200 \mathrm{~W}$,
$0.8 \mathrm{~m} / \mathrm{s}$
3 bar
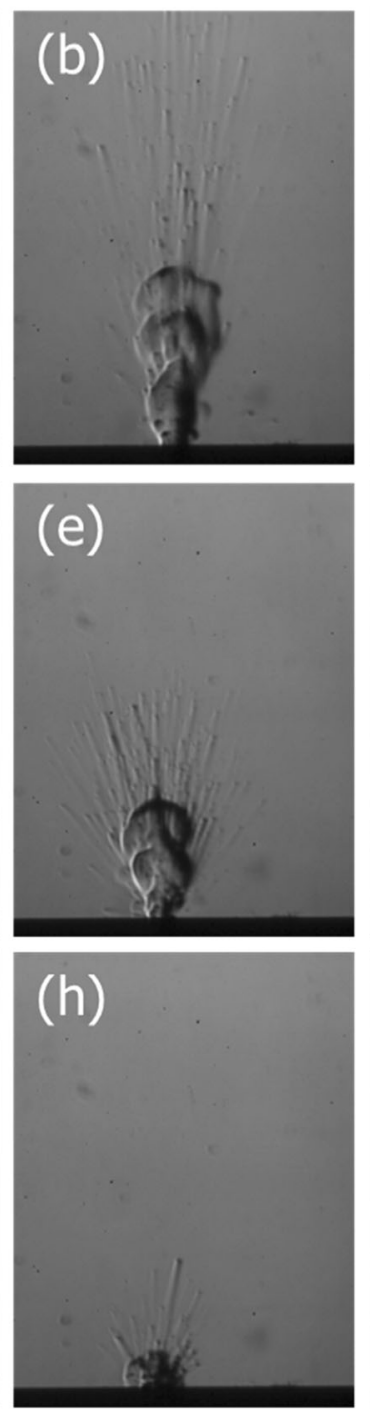

5 bar
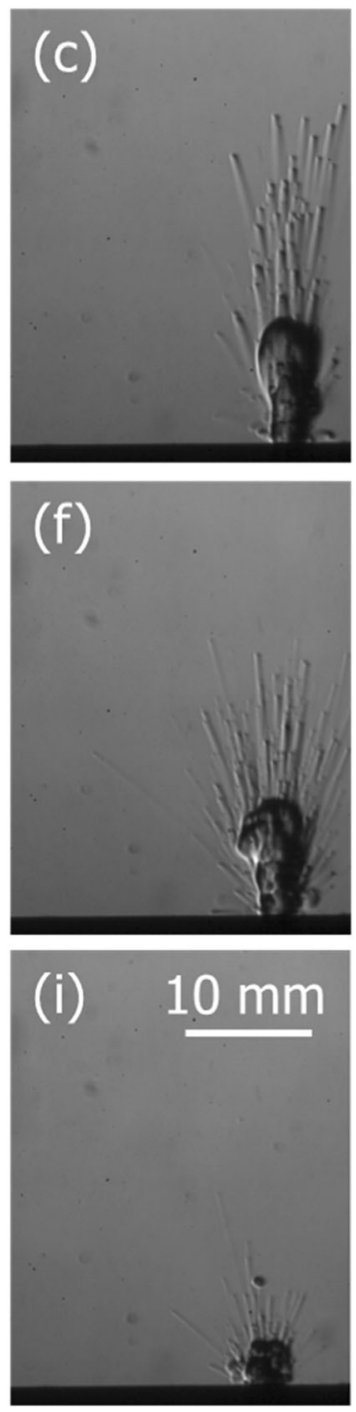
is constrained closer to the powder bed due to the lower velocity of the laser plume from the melt pool. This effect is less pronounced at $200 \mathrm{~W}$ because the laser plume is angled backwards with respect to the laser scan direction.

The schlieren features arise from refractive index gradients due to temperature, pressure and concentration gradients in the shielding gas. However, the schlieren system sensitivity to pressure and concentration gradients across the thermal plume is relatively small compared to the sensitivity to the temperature gradients. The sensitivity of the schlieren system was constant for all the measurements in Fig. 4. Therefore, the darker schlieren features confirm an increase in the temperature of the plume as the pressure increases, as inferred from direct imaging of the plasma in Figs. 2 and 3.

The left-hand column of Fig. 5 and Video Fig. 5 shows the laser spot scanning from right to left for a single track. The results were recorded with the same schlieren sensitivity as in Fig. 4. The images in Fig. 5 were taken with the laser spot at the same distance from the start of the scan line $(8 \mathrm{~mm})$ so as to compare the evolution of the thermal plume. At $200 \mathrm{~W}$, the laser beam traverses less of the thermal plume than at $100 \mathrm{~W}$, due to its higher scan speed and because the laser plume is angled backwards with respect to the laser scan direction.

Figure 6 shows cross-sections of the single track scans. In general, the penetration depth decreases as the pressure increases at each laser setting, which suggests that the physical processes that generate the keyhole are weaker at higher pressure. The penetration at $200 \mathrm{~W}$ at 5 bar is similar to that for $100 \mathrm{~W}$ at 1 bar. These points are discussed further in Section 4.

Figure 7 and Video Fig. 7 show direct imaging of island scans recorded with increasing pressure in argon. The first scanned line of the island is equivalent to the single track scans of Fig. 2, and the direction of the laser plume and the particle entrainment are consistent at each laser setting and pressure. For subsequent tracks in the island, the laser plume is directed away from the previously melted track. The extent of denudation in the first layer can be significant, as the effects of adjacent tracks accumulate and the temperature of the powder bed increases. However, its effect should not be over-exaggerated: the first layer is relatively thin and is spread directly on to the coupon. It has been shown that the change in the powder layer thickness between layers is described by a geometric series and that in the
Fig. 5 High-speed schlieren images at 5 bar during single track scans from right to left when the laser had scanned $8 \mathrm{~mm}$ on the powder bed surface. The schlieren sensitivity is the same as in Fig. 4. The incident laser beam traverses more of the thermal plume of heated gas and metal vapour produced by the melt pool at $200 \mathrm{~W}$ than at $100 \mathrm{~W}$; the thermal plume rises faster in helium

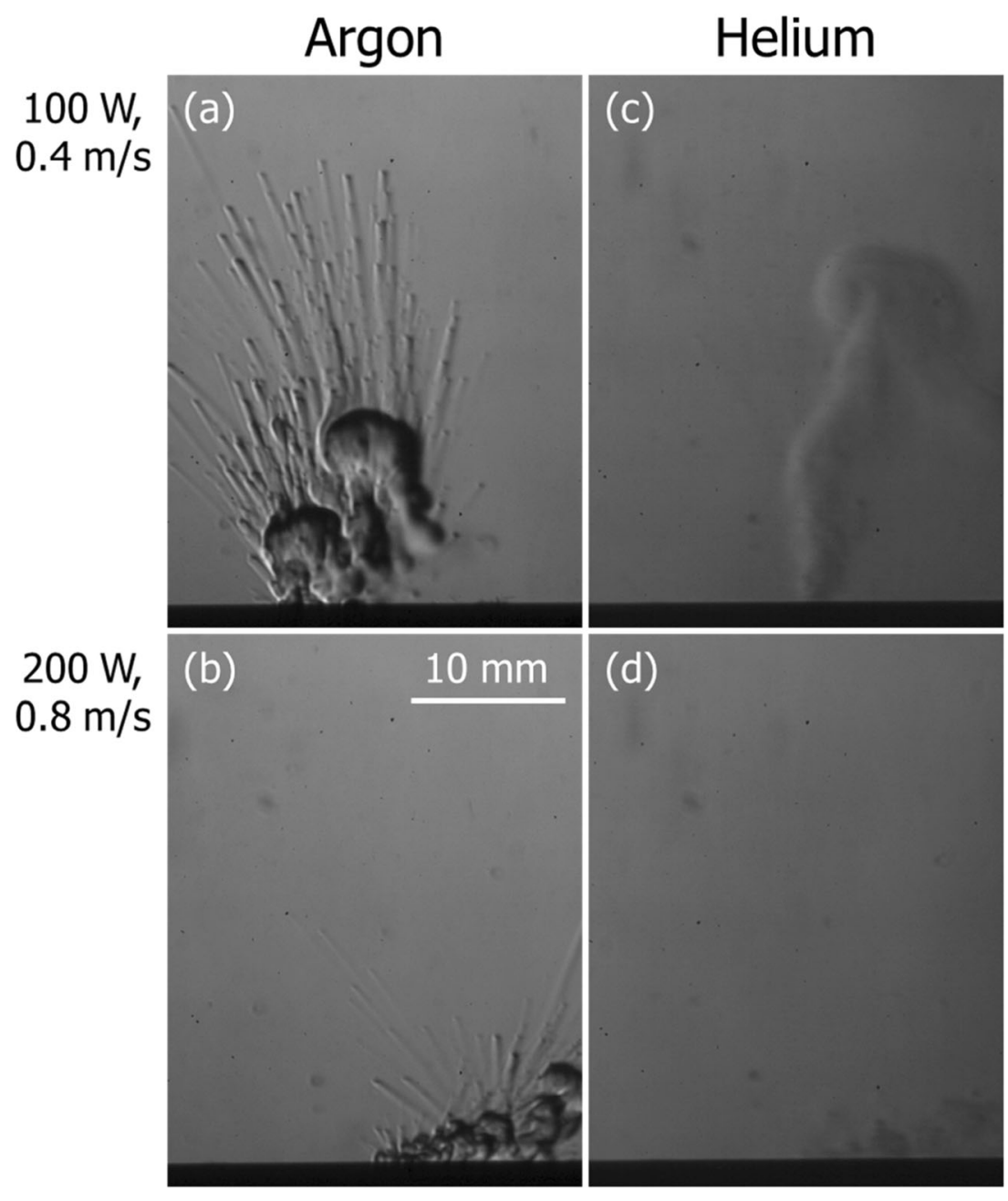


Fig. 6 Cross-sections for single track scans at the pressures and process settings (laser power and scan speed) indicated. The penetration depth decreases with an increase in pressure

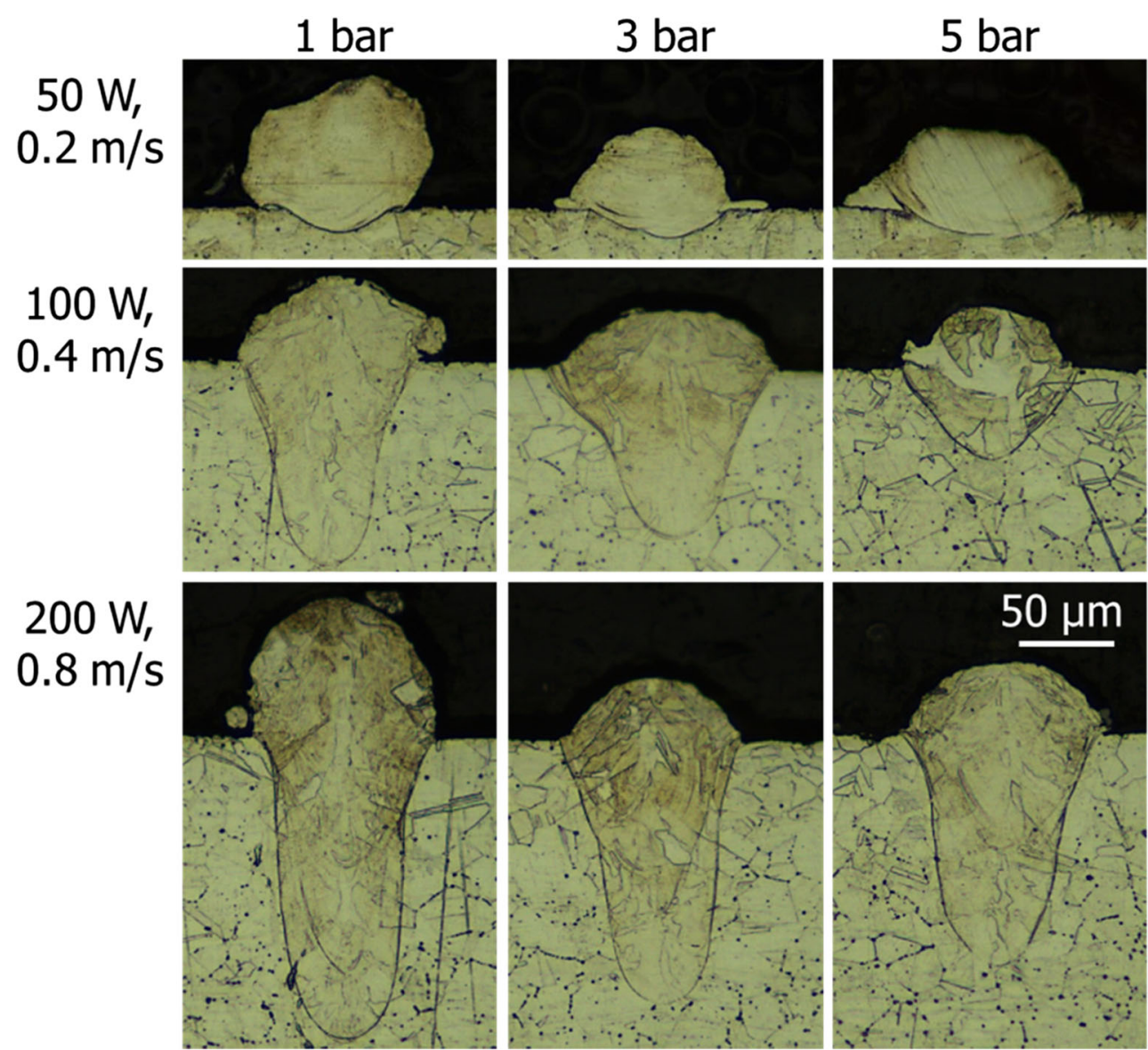

steady state build after 6 to 10 layers the denudation is less severe due to the increased powder layer thickness [9] and the surface roughness of previously built layers [4].
Some denudation is still evident at 5 bar, which causes the laser spot to interact with different amounts of powder as the island scan progresses. The production of plasma and spatter
1 bar

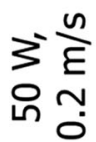

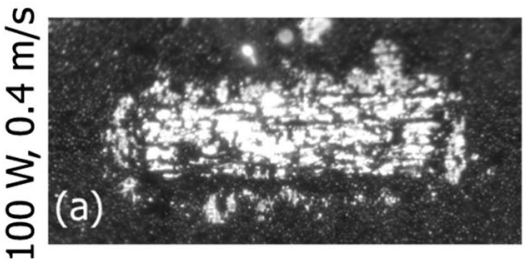

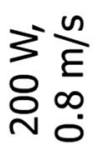

Fig. 7 High-speed images for top views of the powder bed in argon when scanning rectangular islands at the pressures and process settings indicated. Denudation is reduced as the pressure increases at a laser
3 bar

5 bar
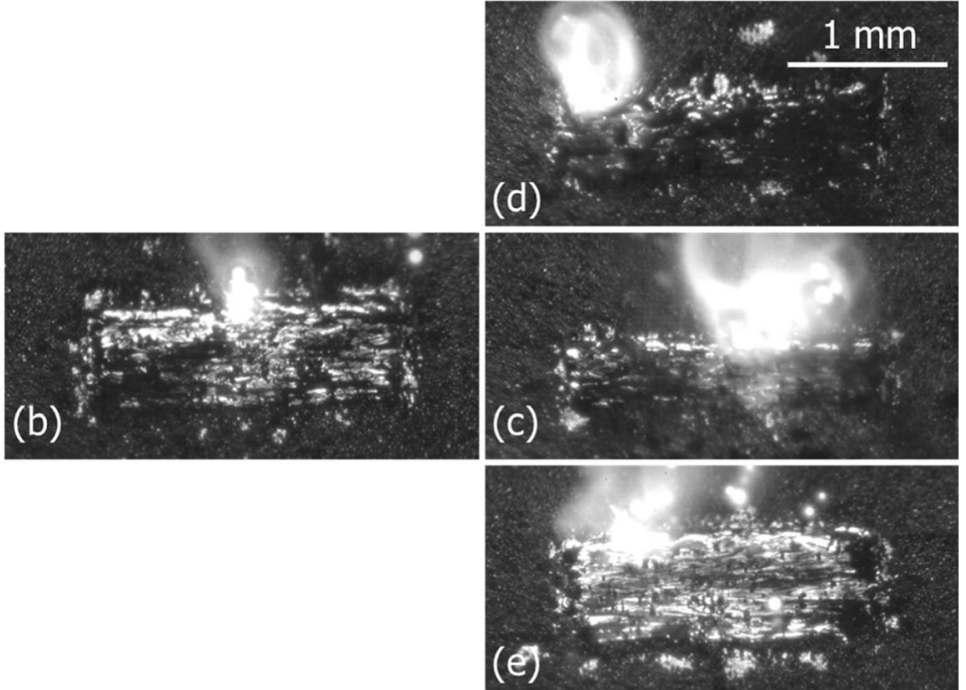

power of $100 \mathrm{~W}$ and scan speed of $0.4 \mathrm{~m} / \mathrm{s}$; the formation of plasma and spatter increases as the laser power and scan speed are increased at 5 bar 
1 bar
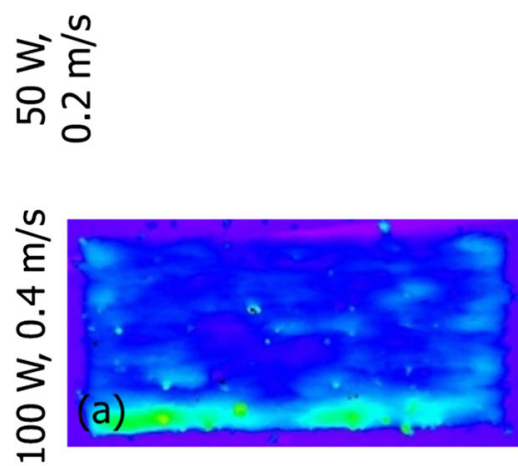

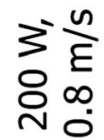

3 bar
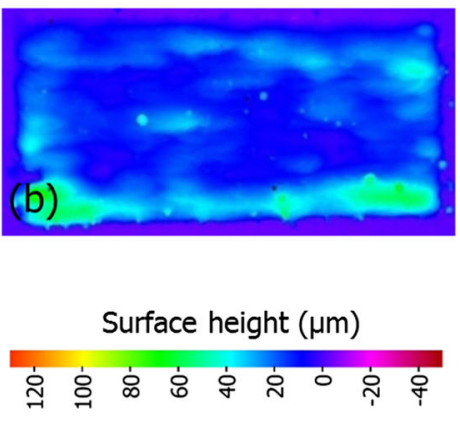

5 bar
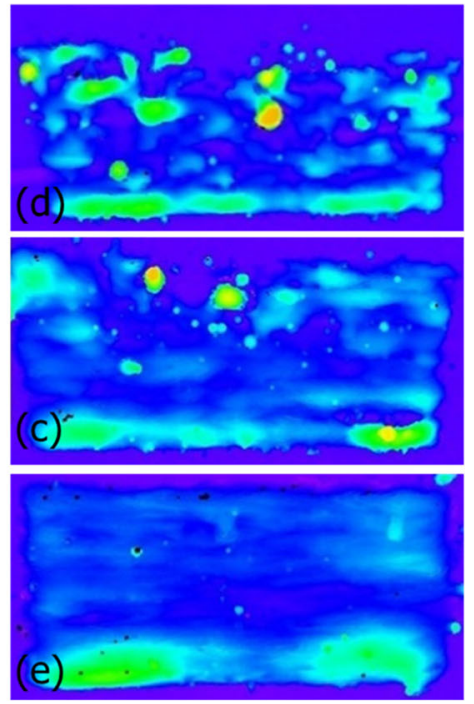

Fig. 8 Surface height maps for the island scans of Fig. 7. The first track of each island is at the bottom of the image

is greatest when more powder is present, indicating that the plasma primarily consists of ionised metal vapour rather than ionised argon, and that variations in the amount of powder incorporated into the melt pool cause melt pool instabilities which contribute to spatter.

Figure 8 shows the build height relative to the build plate for the island scans shown in Fig. 7. The first track of each scan is much higher than the subsequent ones because powder is entrained towards the track from all directions. For the subsequent tracks, the powder denudation significantly reduces the mass consolidated into it. The height of the subsequent tracks at 3 bar, Fig. 8b, are higher on average compared to 1 bar, Fig. 8a, showing the effect of reduced denudation. Although the denudation at 5 bar was observed from the videos to be lower still, the increased interaction between the laser and the laser plume significantly disrupts the process. In general, the smoothness and continuity of the layer worsened with an increase in pressure at a given process setting, but improved with an increase in laser power and scan speed at high pressure.

The islands produced at 5 bar were sectioned orthogonal to the laser scan direction, left-hand column of Fig. 9. Adjacent laser scan lines progress from left to right in the images. As seen in Fig. 7, none of the builds at 5 bar in argon produced a stable build due to the increase in spatter from the melt pool and plasma in the laser plume. The build and penetration depth are greatest for the first track of each island and are consistent with that observed in Fig. 6 at 5 bar. As the island progresses, the penetration depth stabilises but at a decreased value: the change in the shape of the melt pool cross-section is

\section{Argon}
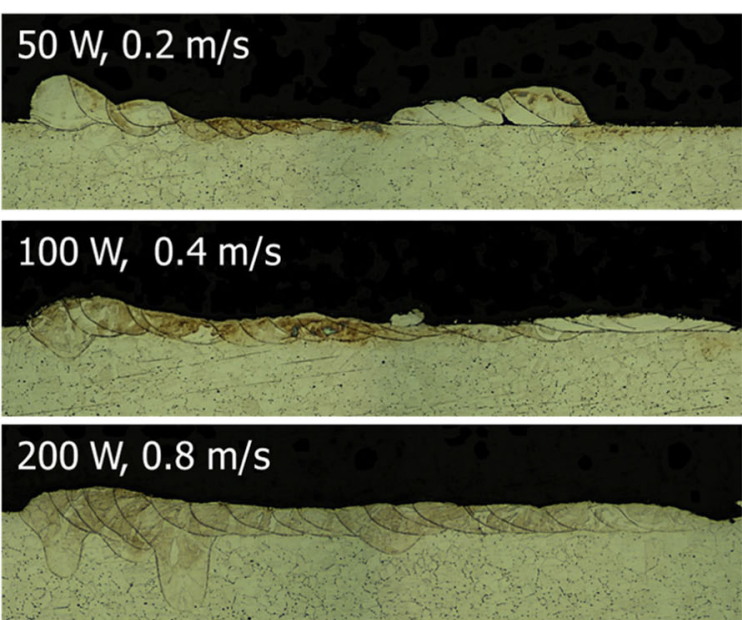

Helium
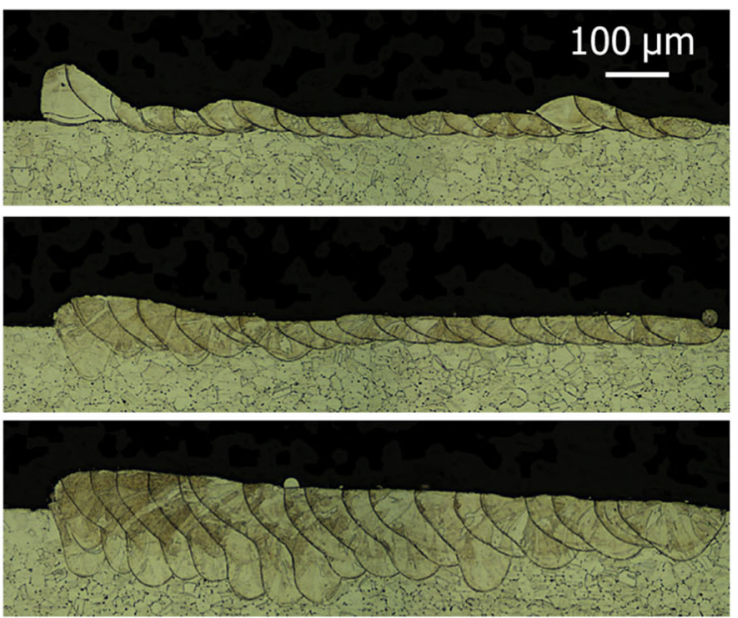

Fig. 9 Cross-sections orthogonal to the laser scan direction for the island scans at 5 bar of Fig. 7 and 10. Adjacent laser scan tracks progress from left to right in the images 
consistent with an increase in laser spot diameter during the island build. This effect is thought to be due to accumulation of metal vapour above the powder bed causing the laser spot to defocus and condensed particulate scattering some of the incident laser beam: all the experiments were recorded without a flow of shielding gas across the powder bed from the flow straightener in order to observe only the laser's interaction with the powder bed.

\subsection{Helium atmosphere}

Figure 10 and Video Fig. 10 show direct imaging of island scans recorded at 1 and 5 bar in helium. The denudation around the islands was greater in helium than in argon at the corresponding pressure, which suggests that the velocity of the laser plume was greater and therefore the induced shielding gas flow and entrainment of powder particles were stronger. For the $100 \mathrm{~W}$ condition, Fig. 10a, b, the denudation decreased as the pressure increased to 5 bar as observed previously for argon. However, in helium, the amount of plasma generated in the laser plume, and the number and size of spatter particles, remained the same at 5 bar compared to 1 bar. These observations indicate that the laser plume in helium was at a lower temperature than at the corresponding pressure in argon. Figure 10c, b, d shows the effect of changing the process setting at 5 bar. Unlike argon, the amount of

\section{1 bar}

plasma, and the amount and size of the spatter, did not increase at higher laser powers and scan speeds: it remained the same in each of the three process conditions. The surface height maps measured from these island scans, Fig. 11, were smoother and more continuous than for argon, and indicate that the process was more stable at high pressure in helium.

The islands produced at 5 bar in helium were sectioned orthogonal to the laser scan direction, right-hand column of Fig. 9. The solidified bead cross-sections of the first track in each island are larger than those at the corresponding process setting in argon, indicating that the energy input was increased. The penetration depth in Fig. 9 was again deeper at the beginning of each island, and stabilised at a decreased value as the island progressed. The decreased penetration began later in the island build than for argon, showing that more time was required in helium for the accumulated metal vapour to defocus the laser beam and for scatter from particulate to reduce its apparent intensity on the powder bed.

Finally, the right-hand column of Fig. 5 shows schlieren imaging for single track scans in the helium atmosphere at 5 bar, recorded with the same schlieren sensitivity as for argon in Figs. 4 and 5. The thermal plume rises faster in helium due to its lower density and kinematic viscosity than for argon. The increased convection in the resulting thermal plume is consistent with the increased number of laser scans required before the accumulated vapour and particulate affected the
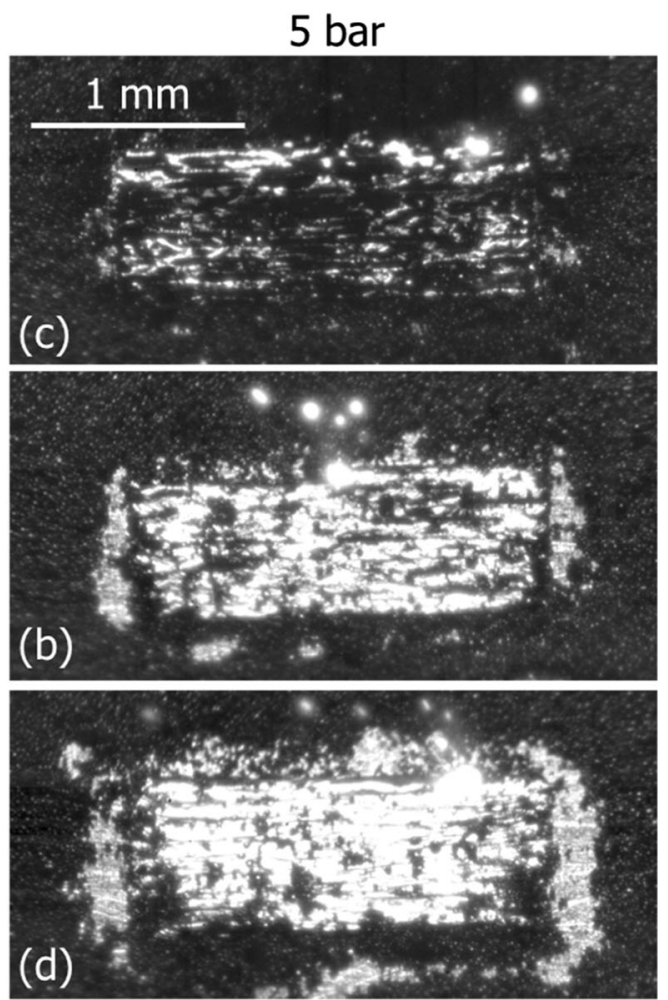

Fig. 10 High-speed images for top views of the powder bed in helium when scanning rectangular islands at the pressures and process settings (laser power and scan speed) indicated. Denudation is larger than at the corresponding setting in argon. Denudation is reduced as the pressure increases at a laser power of $100 \mathrm{~W}$ and scan speed of $0.4 \mathrm{~m} / \mathrm{s}$; the formation of plasma and spatter does not increase at high pressure 
penetration depth of the melt pool. Although the imaged flow features are less dark in helium, it is not possible to infer directly the relative temperature of the thermal plume compared to argon, because the refractive index sensitivity to temperature gradients is lower in helium due to its lower density. However, the absence of significant amounts of ionised metal vapour in the direct imaging results, Fig. 10, enables the inference of a lower temperature plume in helium than in argon.

\section{Discussion}

In an argon environment, particle entrainment (and hence denudation) was reduced at increased ambient pressure, due to the reduction in the speed of the laser plume and the associated reduction in the speed of the induced flow of the shielding gas. However, it was accompanied by more plasma in the laser plume, and by an increase in the number and size of spatter particles ejected from the melt pool, making the process less stable. The increased amount of plasma is due to an observed increase in the temperature of the laser plume at high pressure. The increased internal energy of the plume, combined with an inferred increase in the temperature of the melt pool at high pressure, leads to more powder agglomerates which produce more and larger spatter particles.
The higher temperatures reached in the melt pool are inferred from an increase in vaporisation temperature of the metal at higher pressure and a reduction in energy loss via evaporation. The Clausius-Clapeyron equation describes the coexistence curve on the phase diagram for vaporisation of a material, for which the liquid and vapour phases exist in thermodynamic equilibrium $[5,12,13]$. For stainless steel $316 \mathrm{~L}$, an empirical fit to experimental data in the range 1750 to $5000 \mathrm{~K}$ plot is given by $[14,15]$ :

$\log (P)=11.1183-\frac{18,868}{T}$

with the pressure in $\mathrm{Pa}$. This change in vaporisation temperature with pressure is plotted in Fig. 12 and the vaporisation temperatures at the pressures tested are marked.

For laser welding [16] and PBF [5] experiments at subatmospheric pressures, the penetration depth increases as the pressure is reduced. By analogy, the reduction in penetration observed in Fig. 6 with an increase in pressure is partially explained by this increase in vaporisation temperature. At high pressure, the same incident laser power is absorbed in a shallower melt pool to keep it molten: effectively, more energy is required to create and maintain a weld pool of a given size. A complementary interpretation is that the recoil pressure (the difference between the pressure exerted by the laser plume on the melt pool and the ambient pressure) is reduced at high

\section{1 bar}

\section{$50 \mathrm{~W}$, $0.2 \mathrm{~m} / \mathrm{s}$}

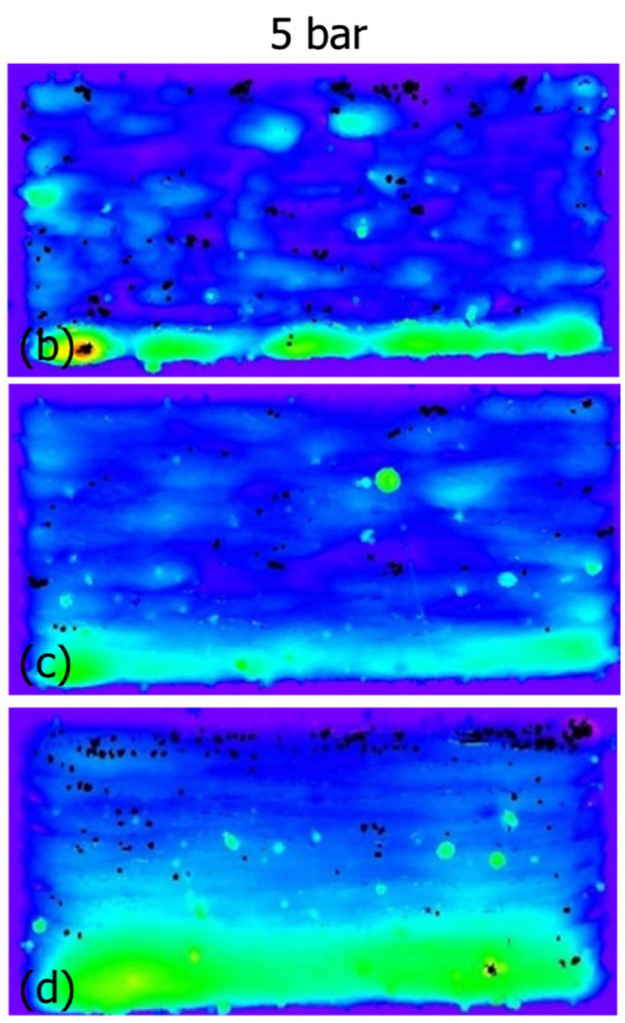

Fig. 11 Surface height maps for the island scans of Fig. 10 


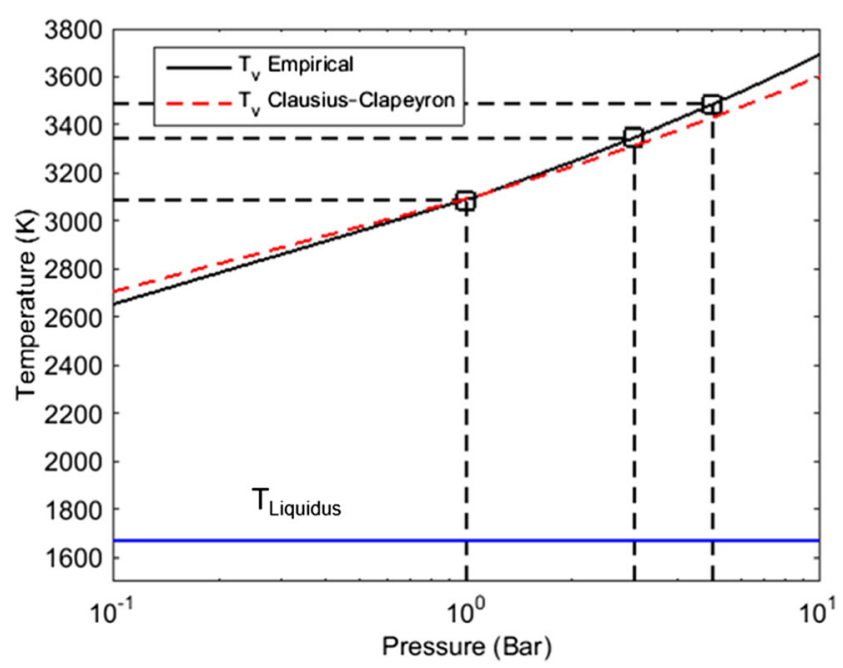

Fig. 12 Plot of vaporisation temperature against pressure for stainless steel 316 L. The empirical line is a plot of Eq. (1) and the ClausiusClapeyron line is taken from [5]. The experimental pressures are marked, for which the corresponding vaporisation temperatures are as follows: $3087 \mathrm{~K}$ (1 bar); 3348 (3 bar) and $3485 \mathrm{~K}$ (5 bar)

pressure, so that more energy is required to overcome ambient pressure to open and then maintain a keyhole.

The second effect that reduces the penetration at high pressure is a decrease in laser energy that reaches the workpiece, due to increased absorption and scattering of the beam by the atmosphere. For a fibre laser operating at a wavelength of $1 \mu \mathrm{m}$ at atmospheric pressure, losses due to Rayleigh scattering (from small particles of condensed metallic atoms with diameter $\sim 100 \mathrm{~nm}$ ) dominate both inverse Bremsstrahlung absorption by the plasma and Mie scattering (from larger agglomerations of condensation particles) $[5,17]$. The effect of each of these scattering and absorption mechanisms increases at high pressure due to the increase in density of the atmosphere. This effect, combined with the increased vaporisation temperature, accounts for the reduction of the penetration depth observed at high pressure.

The temperature increase of the melt pool with an increase in pressure is a complex interaction of many physical processes, several of which vary non-linearly with temperature. As discussed above, the increased vaporisation temperature of the metal and a decrease in energy loss due to reduced evaporation serve to increase its temperature, whilst increased scattering and absorption in the atmosphere at high pressure reduce the laser energy reaching the melt pool. Other physical effects serving to take energy from the melt pool at high pressure include increased metal vaporisation due to the higher temperature of the melt pool and enhanced cooling due to the increased thermal conductivity of the shielding gas. However, the overall result of these complex interactions is an increase in melt pool temperature. It is this increase in melt pool temperature, which occurs as consequence of the high pressure, that is responsible for the increase in spatter.

The increased temperature of the laser plume observed at high pressure is more difficult to analyse analytically or numerically, due to the large number of unknown thermophysical properties at high pressure. However, it is possible to infer information about the ionisation state of the plasma in the laser plume as the pressure, and hence temperature, increases. The first ionisation state of a plasma can be approximated by the Saha equation [18]:

$\frac{N_{\mathrm{e}} N_{\mathrm{i}}}{N_{0}}=\frac{g_{\mathrm{i}} g_{\mathrm{e}}}{g_{0}} \frac{\left(2 \pi m_{\mathrm{e}} k T_{\mathrm{e}}\right)^{3 / 2}}{h^{3}} \exp \left(\frac{-E_{\mathrm{i}}}{k T_{\mathrm{e}}}\right)$

where $N_{\mathrm{e}}, N_{\mathrm{i}}$ and $N_{0}$ are the electron, ion and neutral atom densities, $T_{\mathrm{e}}$ is the electron temperature and constants, $g_{\mathrm{e}}, g_{\mathrm{i}}$ and $g_{0}$ are the degeneracy of the electrons, ionised and neutral states respectively, $E_{\mathrm{i}}$ is the first ionisation potential of the atom, $m_{\mathrm{e}}$ is the electron mass, $k$ is the Boltzmann constant, and $h$ is the Planck constant. For quasi-neutral, weakly ionised plasma $N_{\mathrm{e}}=N_{\mathrm{i}}$ and $N_{0}>>N_{\mathrm{e}}$, and using the equation of state $P=N_{0} k T_{e}$ to approximate $N_{0}$, gives:

$N_{\mathrm{e}}^{2}=\frac{g_{\mathrm{i}} g_{\mathrm{e}}}{g_{0}} \frac{\left(2 \pi m_{\mathrm{e}}\right)^{3 / 2}}{h^{3}}\left(k T_{\mathrm{e}}\right)^{1 / 2} P \exp \left(\frac{-E_{\mathrm{i}}}{k T_{\mathrm{e}}}\right)$

For stainless steel, the metal vapour is predominantly iron vapour ( $\sim 66 \%$ by mass of the powder) but also contains some of the alloying elements, including chromium $(\sim 17 \%)$, nickel $(\sim 12 \%)$ and manganese $(\sim 2 \%)$. Selective vaporisation during laser processing of a metallic alloys may lead to slightly different vaporisation rates of the alloying elements, and hence composition of the plasma. At 1 bar, the laser plume has an expected temperature of $\sim 6000 \mathrm{~K}$, primarily depending on the laser power [4], which is supported by experimental data for the electron density in the laser plume above a laser key-hole weld in iron [18]. There is no comparable experimental data available at high pressure. The electron density for iron given by Eq. 3 is plotted against pressure in Fig. 13a for the atomic constants given in Table 1. Clearly, the temperature varies with position in the laser plume, and two values are included in the figure: $6000 \mathrm{~K}$ corresponding to a typical temperature and $8000 \mathrm{~K}$ to a high estimate of the increased temperature at 5 bar. From Eq. (3) at $6000 \mathrm{~K}$, the degree of ionisation $\left(N_{\mathrm{e}} / N_{0}\right)$ for iron is $2.3 \%$ and for argon is only $0.002 \%$. Even if the electron temperature in the plasma at 5 bar rises to as much as $8000 \mathrm{~K}$, the degree of ionisation of argon is still only $0.09 \%$ which supports the observation that the plasma at high pressure was predominantly ionised metal vapour. The threshold for the optical breakdown of gases decreases at high pressure, but its value for argon at 5 bar is $\sim 3 \times 10^{9} \mathrm{~W} / \mathrm{cm}^{2}$ for a laser wavelength of $1064 \mathrm{~nm}$ [19] which is significantly higher than the highest value in our experiments $\left(1 \times 10^{6} \mathrm{~W} / \mathrm{cm}^{2}\right.$ at $200 \mathrm{~W}$ for a $50-\mu \mathrm{m}$-diameter laser spot). Figure $13 \mathrm{~b}$ shows that the degree of ionisation actually decreases as the pressure increases. However, the overall number of ions increases, as shown in Fig. 13a and observed experimentally. 


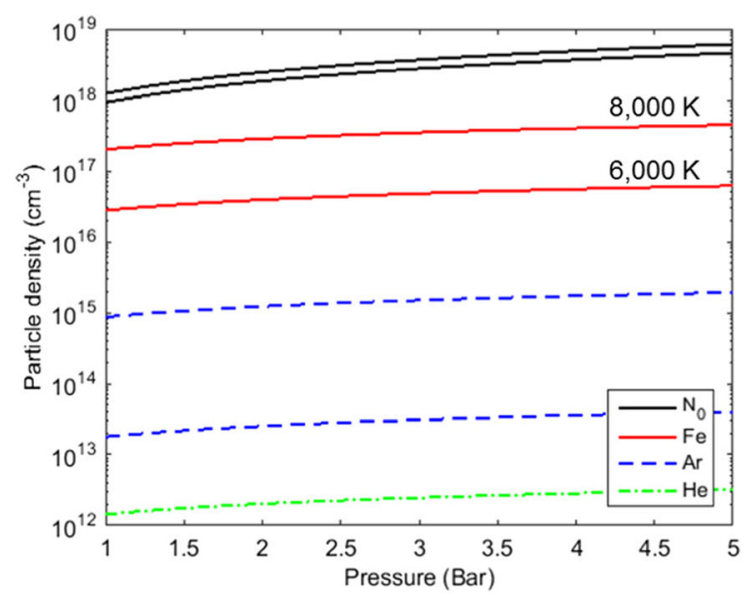

(a)

Fig. 13 a Plot of electron density against pressure for a weak plasma of iron, argon and helium from Eq. (3) using the atomic values in Table 1. For each element, the upper and lower lines correspond to $T_{\mathrm{e}}$ of $6000 \mathrm{~K}$

Increased thermal ionisation of the metal vapour contributes to the increased plasma in the laser plume, due to the increased temperature of the melt pool and laser plume. It is conceivable that as seed electrons are present within the laser's focal volume, cascade photoionisation processes add to the overall degree of ionisation of the metal vapour. This effect would explain the large increase in plasma observed when spatter was expelled upwards, in the direction of the laser beam, compared to that observed during backwards ejection of the laser plume. Theoretical analyses suggest that the intensity threshold for cascade photoionisation is inversely proportional to the pressure squared [20], while competing processes such as recombination are less favourable within the highenergy argon plume.

Denudation around the melt track was always greater in helium than in argon at the corresponding pressure and process setting. The drag force on an entrained powder particle (due to the flow induced in the shielding gas by the laser plume) is proportional to the density of the fluid and the square of its velocity. Hence, the velocity of the laser plume in helium must be greater than in argon. The denudation decreased at high pressure due to a reduction in the laser plume velocity, but with no increase in ionisation of the metal vapour or spatter from the melt pool. The thermal conductivity of helium is approximately ten times that of argon across a range of temperatures, effectively conducting the heat away and preventing the formation of plasma. Any ionisation of helium

Table 1 Atomic constants for atoms and ions of interest [21], used in Eq. (3) and Fig. 13. Note $g_{\mathrm{e}}=2$

\begin{tabular}{lrrc}
\hline & $g_{\mathrm{i}}$ & $g_{\mathrm{o}}$ & \multicolumn{1}{l}{$E_{\mathrm{i}}(\mathrm{eV})$} \\
\hline Iron & 30 & 25 & 7.9024678 \\
Argon & 2 & 1 & 15.7596112 \\
Helium & 2 & 1 & 24.587387936 \\
\hline
\end{tabular}

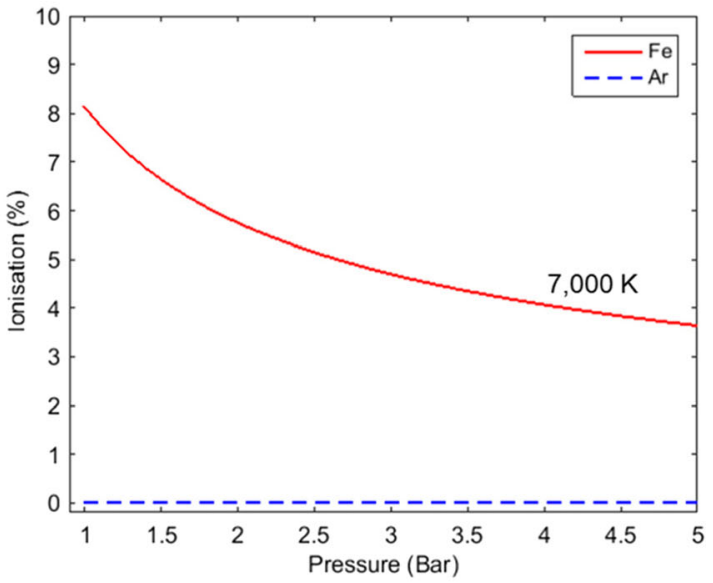

(b)

and $8000 \mathrm{~K}$, respectively. For helium, $N_{\mathrm{e}}<10^{12}$ at $6000 \mathrm{~K}$. b Plot of the degree of ionisation for iron and $\operatorname{argon}$ at $T_{\mathrm{e}}=7000 \mathrm{~K}$

is even less than argon, due to its higher ionisation potential, Fig. 13a, and the optical breakdown intensity threshold is even higher [19]. The thermal plume rises faster in helium due to its lower density and kinematic viscosity, assisting with the convective transport of metal vapour and plasma away from the melt pool. Hence, more energy arrived at the metal surface, resulting in a larger melt bead at the corresponding process setting than in argon. However, even so, the accumulation of vapour and particulate above the powder bed did eventually cause defocus of the laser beam and a reduction in the apparent laser intensity reaching the powder bed, causing the penetration to be reduced in the island scans.

Although denudation was reduced at high pressure, the increased ionisation of metal vapour and spatter from the melt pool degraded the islands in argon: no advantage in using high pressure was identified, at least for the conditions investigated. The helium atmosphere mitigated the negative effects of plasma and spatter to some extent. There is a similarity in denudation and penetration achieved in argon at $1 \mathrm{bar}, 100 \mathrm{~W}$ and $0.4 \mathrm{~m} / \mathrm{s}$ and in helium at $5 \mathrm{bar}, 200 \mathrm{~W}$ and $0.8 \mathrm{~m} / \mathrm{s}$ with a comparable smoothness and continuity of the built layer. As discussed above, this effect can be attributed to a reduction in recoil pressure at 5 bar, resulting in lower kinetic energy in the melt pool and more stable flow. Therefore, a high-pressure atmosphere could potentially enable an increase in processing speed, provided that the metal vapour generated is extracted to prevent laser defocus and excess plasma generation. Alternatively, increasing the pressure can increase the melt pool temperature for like-for-like parameters, meaning that full consolidation could be achieved at a lower energy density. The advantages of high pressure in helium at the conditions tested would not appear to be sufficiently decisive to recommend the additional complexity and expense required in the PBF system. However, the enhanced heat transfer observed 
when using helium certainly warrants further study on the potential benefits of gases and mixtures beyond pure argon. The effects of helium or mixtures with other gases on material properties (such as hardness and microstructure due to refined grain structure with rapid cooling) and fluid-particle interactions would also need to be considered.

\section{Conclusions}

The entrainment of powder particles in laser powder bed fusion can be reduced by increasing the ambient pressure, which in principle reduces the associate effects of powder denudation and porosity. However in argon, the associated increase in the temperature of the melt pool and the laser plume produced more spatter and ionised metal vapour: the smoothness and continuity of built layers was degraded. Due to excess plasma formation, no advantage in using high pressure was identified. In helium, the plasma and spatter were limited by its higher thermal conductivity and diffusivity, and a comparable smoothness and continuity of built layers was achieved to that in argon at atmospheric pressure but at an increased process speed.

Acknowledgements The authors are grateful to Jolyon Cleaves of Vision Research for use of the Phantom V2512 high-speed camera, and to Toby Scrivener of Laser 2000 (UK) Ltd. for use of the Lumencor SOLA SM light engine.

Funding information This work was supported by the Engineering and Physical Sciences Research Council (Grant number EP/K030884/1).

Open Access This article is distributed under the terms of the Creative Commons Attribution 4.0 International License (http:// creativecommons.org/licenses/by/4.0/), which permits unrestricted use, distribution, and reproduction in any medium, provided you give appropriate credit to the original author(s) and the source, provide a link to the Creative Commons license, and indicate if changes were made.

Publisher's Note Springer Nature remains neutral with regard to jurisdictional claims in published maps and institutional affiliations.

\section{References}

1. Gibson I, Rosen DW, Stucker B (2010) Additive manufacturing technologies. Springer, Boston

2. Matthews MJ, Guss G, Khairallah SA, Rubenchik AM, Depond PJ, King WE (2016) Denudation of metal powder layers in laser powder bed fusion processes. Acta Mater 114:33-42

3. Zhao C, Fezzaa K, Cunningham RW, Wen H, De Carlo F, Chen L, Rollett AD, Sun T (2017) Real-time monitoring of laser powder bed fusion process using high-speed X-ray imaging and diffraction. Sci Rep 7:3602

4. Bidare P, Bitharas I, Ward M, Attallah MM, Moore AJ (2018) Fluid and particle dynamics in laser powder bed fusion. Acta Mater 142: 107-120

5. Bidare P, Bitharas I, Ward RM, Attallah MM, Moore AJ (2018) Laser powder bed fusion at sub-atmospheric pressures, 130-131 65-72. Int J Mach Tools Manuf

6. Simonelli M, Tuck C, Aboulkhair NT, Maskery I, Ashcroft I, Wildman RD, Hague R (2015) A study on the laser spatter and the oxidation reactions during selective laser melting of $316 \mathrm{~L}$ stainless steel, Al-Si10-Mg, and Ti-6Al-4V. Metall Mater Trans A 46: 3842-3851

7. Wang X, Read N, Carter LN, Ward RM, Attallah MM (2016) Defect formation and its mitigation in selective laser melting of high $\gamma^{\prime}$ Ni-base superalloys in Superalloys 2016. In: Hardy RK et al (eds) . Wiley, pp 351-358

8. Gieseke M, Noelke C, Kaierle S, Wesling V, Haferkamp H (2013) Selective laser melting of magnesium and magnesium alloys. In: Proceedings of Magnesium Technology 2013, The Minerals, Metals and Materials Society. Springer

9. Bidare P, Maier RRJ, Beck RJ, Shephard JD, Moore AJ (2017) An open-architecture metal powder bed fusion system for in-situ process measurements. Addit Manuf 16:177-185

10. Renishaw plc (2017) Data sheet: SS 316L-0407 powder for additive manufacturing

11. Bitharas I, Campbell SW, Galloway AM, McPherson NA, Moore AJ (2016) Visualisation of alternating shielding gas flow in GTAW. Mater Des 91:424-431

12. Zhang BC, Liao HL, Coddet C (2013) Microstructure evolution and density behavior of CP Ti parts elaborated by self-developed vacuum selective laser melting system. Appl Surf Sci 279:310-316

13. Masmoudi A, Bolot R, Coddet C (2015) Investigation of the laserpowder-atmosphere interaction zone during the selective laser melting process. J Mater Process Technol 225:122-132

14. Kim CS (1975) Thermophysical properties of stainless steels, ANL75-55. Argonne National Laboratory, USA

15. International Atomic Energy Agency (IAEA) (2008) Thermophysical properties of materials for nuclear engineering: a tutorial and collection of data

16. Katayama S, Yohei A, Mizutani M, Kawahito Y (2011) Development of deep penetration welding technology with high brightness laser under vacuum. In: Proceedings Lasers in Manufacturing (LIM), Phys Procedia 12 Part A 75-80

17. Kawahito Y, Kinoshita K, Matsumoto N, Katayama S (2009) Visualization of refraction and attenuation of near-infrared laser beam due to laser-induced plume. J Laser Appl 21(2):96-101

18. Verwaerde A, Fabbro R, Deshors G (1995) Experimental study of continuous CO2-laser welding at subatmospheric pressures. J Appl Phys 78(5):2981-2984

19. Panarella E (1974) Theory of laser-induced gas ionization. Found Phys 4(2):227-259

20. Morgan CG (1975) Laser-induced breakdown of gases. Rep Prog Phys 38:621-665

21. Han W (2004) Computational and experimental investigations of laser drilling and welding for microelectronic packaging, $\mathrm{PhD}$ thesis, Worcester Polytechnic Institute 